\title{
Lange Briefe. Überlegungen zu einem Medienformat am Beispiel der Briefe Dorothea Schlegels
}

\author{
Mit einem unveröffentlichten Brief Dorothea Schlegels im \\ Anhang
}

Der folgende Beitrag beschäftigt sich mit dem Status des Briefes zwischen Ereignis und Objekt und fragt nach Frequenz oder Permanenz von Briefen. Vor allem das Problem der Frequenz steht im Zentrum der folgenden Überlegungen zum langen Brief. Es ergibt sich aus dem Umstand, dass lange Privatbriefe als Gaben verstanden werden können, die als Gegengaben wiederum lange Briefe einfordern. Ein solcher Austausch aber lässt sich nicht beliebig steigern, etwa durch immer längere Briefe, und auch nicht beliebig lange durchhalten. Irgendwann geht auch der eifrigsten Schreiberin die Luft aus. Insofern sind lange Briefe anstrengende Gaben, die ihre Empfänger in Verlegenheit versetzen können. In Gestalt der Länge ist gleichsam ein kleiner Giftpfeil in sie eingewickelt.

Der Beitrag wählt für die Analyse der spezifischen Zeitlichkeit und des prekären Gabencharakters langer Briefe eine medienwissenschaftliche Perspektive, deren Kompatibilität mit kulturanthropologischen Herangehensweisen über den Begriff der Gabe auf der Hand liegt, hier aber nicht weiter ausgeführt werden kann. Eine medienwissenschaftliche Perspektive bietet sich für das Problem der langen Briefe vor allem darum an, weil sie mit statistischen Datenerhebungen kompatibel ist. Mit ihrer Hilfe lässt sich die Stellung des langen Briefs zwischen Nähe und Distanz dann nicht zuletzt auf der Grundlage großer Datenmengen beantworten. Die soziale Leistung von Briefen, ihr Beitrag zur sozialen Praxis, wird in dieser Perspektive zu einem Effekt der Menge an Zeichen, aus denen er besteht.

Die Überlegungen beginnen mit einer Bestimmung des ,langen Briefs‘. Ihr liegt ein quantitatives und relationales Verständnis des Phänomens der Brieflänge zu Grunde (Teil 1). Sodann geht es am Beispiel der Briefe Dorothea Schlegels um eine Typologie der Gründe für das Verfassen langer Briefe (Teil 2). In einem dritten Schritt werden in vergleichender Absicht statistische Daten zu den Briefwechseln Friedrich und Dorothea Schlegels präsentiert und ausgewertet (Teil 3). Im vierten und letzten Teil wird schließlich auf der Grundlage der erarbeiteten Begriffe

Ulrich Breuer und Anke Lindemann, Johannes Gutenberg-Universität Mainz

Ә Open Access. ( 2021 Ulrich Breuer, Anke Lindemann, publiziert von De Gruyter (c))BY-NC-ND Dieses Werk ist lizenziert unter der Creative Commons Attribution-Non-Commercial-NoDerivatives 4.0 International Lizenz. https://doi.org/10.1515/9783110712568-010 
und Erkenntnisse in exemplarischer Absicht ein langer Brief Dorothea Schlegels analysiert (Teil 4).

\section{Was sind lange Briefe?}

Der lange Brief ist eine brieftheoretisch bisher unterbelichtete Form des Briefs. In der Briefforschung taucht er bislang nur am Rande auf. So rechnet etwa Peter Bürgel in einem sowohl medienwissenschaftlich als auch anthropologisch angelegten Beitrag aus den späten 70er Jahren in kulturkritischer Perspektive die abnehmende „Länge des Einzelschreibens“ zu den klaren Indizien für den durch Presse, Telegraph und Postkarte eingeläuteten „Niedergang des B.[rief]s“ nach 1848. ${ }^{1}$ Man kann das als eine erste Hypothese zur Geschichte des langen Briefs lesen, die dann empirisch zu verifizieren wäre.

Dass freilich auch ein halbes Jahrhundert nach 1848 der lange Brief noch zu den gängigen Optionen eines Briefschreibers gehörte, zumindest zu dessen literarischer Figuration, lässt sich Rilkes Gedicht Herbsttag entnehmen. Es entstand am 21.9.1902 in Paris, einem notorischen Ort für Exorbitantes aller Art. In dem Gedicht ist der lange Brief Teil einer Stimmung, also eines Verschwimmens von Subjekt/Objekt-Differenzen. Man könnte die Stimmung in Herbsttag als diejenige eines Solitärs im Herbst seines Lebens bezeichnen. Evoziert wird sie unter anderem mit den bekannten Versen:

Wer jetzt allein ist, wird es lange bleiben, wird wachen, lesen, lange Briefe schreiben. ${ }^{2}$

Das Gedicht stellt einen intrinsischen Zusammenhang her zwischen der Stimmung der Einsamkeit, ihrer Dauer und dem Medienformat des langen Briefes. Dieser Zusammenhang, besonders der Hinweis auf das Alleinsein, kann stutzig machen, denn der Brief wird seit der Antike als dialogische Gattung und Gesprächsersatz verstanden. ${ }^{3}$ Seit dem 18. Jahrhundert nutzt man ihn sogar ,als funktionales Äquivalent des geselligen Betragens“.. ${ }^{4}$ In grundlegender Weise ist es dabei der Wechsel, der Austausch von Schriftstücken, der einen Brief zum Brief macht. Der dem Brief zugeordnete Mediengebrauch heißt daher ,Korrespondieren'. Er bezeichnet eine Beziehungsform. Damit der Austausch, der diese Beziehungsform konstituiert,

1 Bürgel 1979, 28.

2 Rilke 1996, 281.

3 Nickisch 1991, 4.

4 Vellusig 2000, 155. 
gut funktioniert, damit es gewissermaßen wechselseitig flutscht, muss der Brief so sein, wie er heißt, nämlich brevis, also kurz. Seine Kürze steht bekanntlich in engem Zusammenhang mit seinem Informationscharakter, denn als „brevis (libellus)“ ${ }^{5}$ wurden bis in die Frühe Neuzeit hinein hauptsächlich Nachrichten verstanden. Tauscht man Nachrichten aus und stellt sich dabei selbst mit dar, so sind die Bedingungen für den Privatbrief erfüllt. Er ist auf den privaten Wechsel von Informationen angelegt und lässt sich nur so lange reibungslos fortsetzen, wie die Menge der jeweils übermittelten Informationen begrenzt wird. Seine informationsbedingte Kürze unterscheidet den Brief von der Epistel, ${ }^{6}$ die als apostolisches oder öffentliches Mahnschreiben und natürlich auch als poetische Gattung nicht auf Wechselseitigkeit angelegt und daher auch keinen Längenbeschränkungen unterworfen ist. Metonymisch und umgangssprachlich kann daher der Empfänger eines langen Briefes auch von einer ,wahren Epistel` sprechen. Auf der anderen Seite der Skala steht der kurze Brief, der, zum Billett geschrumpft, ebenfalls nicht so recht zum Austausch taugt. Hier ist die Informationsmenge gewissermaßen zu gering und überdies nicht narrativ aufgearbeitet.

Drückt man den Punkt, auf den es hier ankommt, mit Hilfe der Differenz von Medium und Form aus, dann widerstrebt der lange Brief dem Medium durch seine Form. Er ist in medientheoretischer Perspektive eine Störung und er ist es gerade dann, wenn er die Beziehung zwischen den Briefpartnern als besonders großzügige Gabe massiv intensiviert. Man könnte diese Störung näher als drohende Blockade der Antwort durch ,information overload' charakterisieren, wobei der lange Brief, aufgrund des für ihn zu betreibenden Zeitaufwands für Schreiber und Leserin, strukturell in die Nähe des ausbleibenden Briefes und damit gefährlich nahe an den Abbruch, zumindest aber an eine Unterbrechung des regelmäßigen Wechsels rückt. Es kommt hinzu, dass der Briefpartner bei verzögerter Antwort seinerseits mit einem weiteren Schreiben zu reagieren droht, so dass die wechselseitige Taktung des Briefwechsels, der zu seinen Möglichkeitsbedingungen gehört, Gefahr läuft, ins Stottern zu geraten und aus dem Rhythmus gebracht zu werden. Die mögliche Blockade der Korrespondenz, die der lange Brief durch seine Informationsmasse einseitig ins Spiel bringen kann, droht das dialogische Medium Brief in ein monologisches $\mathrm{zu}$ verwandeln und es dadurch um seine Leistung $\mathrm{zu}$ bringen.

Medienwissenschaftler werden darauf mit Neugierde reagieren. Denn zum einen sind Medien generell schwer zu erkennen und verraten sich am ehesten dann, wenn sie in ihrer Funktion gestört werden. Zum anderen ist ,in der Veränderung

5 Nickisch 1991, 22.

6 Vgl. Kemper 1997. 
von Größenverhältnissen“ das eigentliche Thema der Medienwissenschaft gesehen und empfohlen worden, die Medienwissenschaft als „Skalierungsanalyse“ $\mathrm{zu}$ reformulieren. ${ }^{7}$ Eben das macht den langen Brief für die Brieftheorie interessant. Er stellt einen Grenzwert des Mediums Brief dar, weil er das Hin und Her, den wechselseitigen Austausch, dadurch intensiviert (wenn etwa auf lange Briefe lange Briefe folgen), dass er ihn zugleich erschwert (denn lange Briefe kosten Zeit und Geld) und bei weiterhin ansteigender Länge (von 10 auf 50, 100 oder gar 1000 Seiten) am Ende auch sistiert. Nähebeziehungen wie diejenigen, die der Brief konstituiert, können durch zu viel Nähe zerstört werden. Sie benötigen offenbar eine Äquidistanz, die auch für eine Kulturanthropologie des Briefes von zentraler Bedeutung ist.

In medientheoretischer Perspektive schlägt sich das Korrespondenzproblem der Äquidistanz unter anderem in dem unscheinbaren Kriterium der Brieflänge nieder. Mit ansteigender Durchschnittslänge scheint, so die hier angesetzte Hypothese, auch der Grad der erstrebten Nähe und mit ihr die Gefahr einer Überhitzung der Korrespondenzbeziehung zuzunehmen, mit abnehmender Länge kühlt diese Beziehung dagegen ab. Trifft das zu, dann können lange Briefe genutzt werden, um eine Korrespondenz als ganze auf den Grad der in ihr erstrebten Nähe, aber auch das Korpus eines langdauernden Briefwechsels auf Phasen der größeren oder geringeren Nähe hin zu profilieren. Werden lange Briefe einseitig präferiert, dann können sie dazu führen, dass Beziehungen verhindert oder bestehende beschädigt, ja zerstört werden. Dazu sei hier nur an Kafkas briefliche Exzesse gegenüber seiner Verlobten Felice Bauer, ${ }^{8}$ aber auch an die monströsen Konvolute erinnert, die Rolf Dieter Brinkmann aus Rom und Olevano Romano an seine damit völlig überforderte Ehefrau Maleen geschickt und aus denen der Rowohlt-Verlag dann den Band Rom, Blicke zusammengestellt hat. ${ }^{9}$

Als Medienformat muss der lange Brief verstärkt auf das Interesse einer Literaturwissenschaft stoßen, die das Format der Literatur für eine relevante Größe hält. ${ }^{10}$ Erschließen lässt es sich nur durch die Zusammenarbeit mehrerer Disziplinen, unter anderem der Literatur- mit den Medien- und den Buchwissenschaften. Im Zentrum entsprechender Forschungsinteressen stehen dabei wie gesagt Skalie-

7 Spoerhase u. Wegmann 2018, 417.

8 Nach Stach $(2015,163)$ erhebt Kafka in dieser Korrespondenz das Medium des Briefs zum Selbstzweck und „überfordert es damit in schrankenloser Weise“. Allerdings relativiert Stach (2015, 163-167) diese Einschätzung dann durch die These, dass Briefe eine Beziehung nicht nur ausdrücken, sondern auch mehr oder weniger suggestiv formen können.

9 Vgl. Brinkmann 1979.

10 Vgl. Spoerhase 2018. 
rungsfragen. ${ }^{11}$ Auch der lange Brief ist ein Skalierungsphänomen. Es setzt voraus, dass er sowohl vom kurzen Brief als auch vom Brief mittlerer Länge hinreichend trennscharf unterschieden werden kann. Das am besten geeignete weil am eindeutigsten objektivierbare Referenzmerkmal dürfte die maschinell leicht erfassbare Anzahl der Zeichen einer Transkription sein. Mit ihr kommt ins Spiel, was Richard M. Meyer in einem frühen und wegweisenden Beitrag zum Problem der Vollständigkeit als „philologische Statistik“12 bezeichnet und als Desiderat einer zeitgemäßen Germanistik ausgewiesen hat.

Es bietet sich vor diesem Hintergrund an, zunächst einen Normbrief (mittlerer Länge) als Mittelwert der Zeichenmenge aller Briefe eines Briefkorpus zu berechnen. Davon ausgehend lassen sich dann diejenigen Briefe in einem gegebenen Korpus, die mehr als die doppelte Zeichenmenge des Normbriefs aufweisen, als lange Briefe definieren. Als kurze Briefe können dann analog diejenigen Briefe definiert werden, welche die Hälfte der Zeichenmenge des Normbriefs nicht erreichen. Damit ist ein heuristischer, je nach Korpus variabler Ausgangswert für den langen Brief gewonnen, der für die Gesamtkorrespondenz zweier Korrespondenzpartner ganz andere Werte ergeben wird als für eine andere Korrespondenz. Der Verzicht darauf, für den langen Brief eine absolute Größe festzulegen, bietet die Chance, jeden einzelnen Korrespondenzpartner oder Gruppen von ihnen schärfer in den Blick zu nehmen oder die Längenverschiebungen im Laufe eines Lebens, während besonderer persönlicher oder politischer Ereignisse etc. zu untersuchen. Insbesondere in Zeiten digitaler Briefeditionen sind weitere Korpusbildungen denkbar wie zum Beispiel das Korpus sämtlicher Briefwechsel einer literarischen Gruppierung wie der Romantik, einer sozialen Formation, einer Epoche, einer Nationalliteratur, etc. Das ermöglicht neue, höchst aufschlussreiche Vergleiche, die dann auch empirisch belastbare Aussagen über Nähebeziehungen erlauben.

Als Materialgrundlage des vorliegenden Beitrags dienen die Briefe Dorothea Schlegels. Das liegt nahe, weil wir uns seit einigen Jahren mit ihrer Korrespondenz und der ihres Mannes beschäftigen. Überdies wurden gerade Dorotheas Briefe auch in der Forschung als besonders lang, oder, wie man am Anfang des zwanzigsten Jahrhunderts abwertend formulierte, als besonders breit wahrgenommen. Verbunden war die abwertende Feststellung der Breite mit dem Vorwurf der Geschwätzigkeit. Er konnte sich nicht zuletzt auf die Autorin selbst berufen. 1811 hatte sie in ihrem Tagebuch festgehalten: „Oft habe ich einen wahren Widerwillen gegen alles Schreiben, dann fliegt mir plötzlich ein wahrer Appetit dazu an. Es ist doch

11 Spoerhase u. Wegmann 2018.

12 Meyer 1907, 7. 
oft weiter nichts, als dass man gern schwatzen möchte [...]." ${ }^{13}$ Die germanistische Forschung des frühen 20. Jahrhunderts hat das gegen sie verwendet. Für Franz Deibel etwa arten die Briefe Dorotheas „nur zu oft in breite Geschwätzigkeit aus, die bis zum Überdruss ihr eigenes Wort, dass eine mässige ökonomische Mitteilung nie ihre Stärke war, zu erhärten sucht. " ${ }^{14}$ Heinrich Finke dagegen stellte ihr Briefwerk immerhin mit demjenigen von Caroline Schlegel-Schelling und Rahel Levin Varnhagen auf eine Stufe, attestierte aber vor allem den späten Briefen Dorotheas in ambivalenter Weise eine zwar „behagliche, oft [aber] auch als unangenehm empfundene Breite“. ${ }^{15}$ Inzwischen ist ein neuer Zugang möglich geworden, der die Geschwätzigkeit im Brief weniger als Charakterfrage, sondern nüchterner als einen Medieneffekt zu begreifen empfiehlt. ${ }^{16}$ Daher soll hier im Ausgang vom quantitativen Kriterium der Brieflänge und des Umgangs mit ihr auch die Frage nach der Qualität der Briefe Dorothea Schlegels neu gestellt und unter Beachtung der historischen Kontexte positiver als bisher beantwortet werden. ${ }^{17}$ Es geht also auch um eine Ehrenrettung. Berufen kann sie sich auf die Stimmen von Zeitgenossen Dorotheas, denen die Freundin vor allem in ihren Wiener Jahren nicht nur besonders liebenswürdig und originell, sondern auch intellektuell brillant, witzig und geistreicher als Friedrich Schlegel erschienen ist. ${ }^{18}$

\section{Warum lange Briefe? Versuch einer Typologie}

Wie kann man die Faktoren, die die Länge von Privatbriefen beeinflussen, kategorisieren ${ }^{19}$ Hier wird vorgeschlagen, die literaturwissenschaftliche Grundunterscheidung zwischen einer produktions-, einer werk- und einer rezeptionsbezoge-

\footnotetext{
13 Schlegel 1881, 121.

14 Deibel 1905, 75.

15 Finke 1918, 87.

16 Vgl. Vellusig 2000, 106.

17 In diese Richtung geht auch Carola Stern, die generell auf die Fähigkeit Dorotheas zu lebhaften Schilderungen und ihren klaren, von Phrasen freien Stil hinwies (Stern 2006, 209) sowie speziell ihre „Brief-Reportagen über Haupt- und Staatsaktionen, Kunsterlebnisse und Reisen“ hervorhebt (Stern 2006, 218). Christina Ujma hat sie als „scharfe Beobachterin“ charakterisiert (Ujma 2001, 47), während Julius H. Schoeps $(2020,25)$ ihren Humor, ihre Neigung zum Übermut und den „ihr eigenen ironisch-spöttischen Tonfall“ unterstreicht. Lange Briefe spielen bei diesen Ehrenrettungen allerdings keine Rolle.

18 Vgl. Stern (2006, 253), die Jean Paul und Justinus Kerner zitiert, sowie Ujma (2001, 53), die Louise Seidler anführt.

19 Wir danken Matthias Emrich für seine Recherchen zum ersten und seine wertvollen Hinweise und Anregungen zum zweiten Abschnitt des Beitrags.
} 
nen Perspektive ${ }^{20} \mathrm{zu}$ nutzen und sie für das Medium Brief als schreiberbezogene, dokumentbezogene und adressatenbezogene $\mathrm{zu}$ respezifizieren. Um die daraus abgeleiteten Faktoren auszuführen, wird fallweise auf Beispiele aus den Briefen Dorothea Schlegels zurückgegriffen.

Zu den schreiberbezogenen Faktoren langer Briefe gehört vor allem die Schreibszene. Zentral ist dabei die ihr zugeordnete Zeitökonomie. Hat man wenig Zeit zum Schreiben, wird der Brief eher kurz und flüchtig ausfallen; gibt es dagegen eine ausgedehnte Mußephase, die sich zudem über mehrere Tage hinzieht, kann er lang werden. Auch eine extern veranlasste zeitliche Ausdehnung der Schreibszene gehört in diesen Zusammenhang. Wenn etwa die Abreise des Überbringers eines Briefes sich verzögert, kann dies zu einem Anschwellen des Briefumfangs führen peu à peu: Noch ein Tag, noch ein Absatz. Relevant ist auch die Häufigkeit, mit der eine Schreibszene auftritt. In der Regel korrespondiert Dorothea beispielsweise nicht mit den Brüdern ihres Ehemannes, doch wenn Friedrich wieder einmal verreist ist, nutzt sie das, um einen intimen und offenen Brief an den Bruder August Wilhelm zu schreiben - der dann prompt auffällig lang ausfällt. ${ }^{21}$

Nicht zuletzt kann auch die anschwellende Ereignisdichte und/oder Ereignisqualität im Umfeld einer Schreibszene die Brieflänge steigern, weil dann die Menge der mitzuteilenden Informationen zunimmt. Das ist etwa bei Reisebriefen, aber auch in Zeiten politischer Umbrüche oder privater Unglücksfälle zu beobachten.

$\mathrm{Zu}$ den dokumentbezogenen Faktoren langer Briefe gehört zum einen der Beschreibstoff. Weil das Papier, je weiter man historisch zurückblickt, ein desto kostspieligeres Gut gewesen ist, will man es in der Regel optimal nutzen und beschreibt es daher so vollständig und dicht wie möglich. Gelegentlich wird dies auch thematisiert. ${ }^{22}$ Die Wahl eines neuen Bogens lässt daher die Brieflänge geradezu zwangsläufig um vier Seiten - und nicht nur um eine oder eine halbe Seite anschwellen. ${ }^{23}$

Dazu wäre eine Untersuchung der Relation von Bogenzahl und Zeichenmenge aufschlussreich. Neben dem Materialwert und seinen Längeneffekten sind aber

20 Fricke 2007, 42-45.

21 Vgl. Dorothea Schlegel an August Wilhelm Schlegel [Wien, Anfang Juni 1811]: „Ich habe schon sehr lange den innigen Wunsch, und ein rechtes Verlangen, Ihnen theurer Freund! einmal recht ausführlich über unsre Lage hier zu schreiben, [...] ich benutze aber jetzt die letzten Tage seiner Abwesenheit, da er in Baden ist [...], um Ihnen einmal recht vertraulich allerlei zu erzählen. [...] um meinem geängstigten Herzen Luft zu machen.“ (Körner 1969, 210-211).

22 Vgl. Patsch 2007, 281.

23 In seiner Satire Orator ineptus spottet etwa Johann Balthasar Schupp über den Hang der Zeitgenossen zu Briefen, bei denen „das gantze Blat von oben biß unten mit Worten angefüllet ist“ (Schupp 1665, A VII ${ }^{\mathrm{V}}$ ). Der Hinweis ist bedeutsam, weil er die Materialität des Mediums anspricht. 
auch die Transportkosten relevant. Ein langer Brief ist umfangreicher als ein kurzer und daher auch teurer. Man muss ihn sich leisten wollen und können. Länge hat also ihren Preis und der lange Brief stellt das performativ auch aus.

$\mathrm{Zu}$ den adressatenbezogenen Faktoren langer Briefe gehört unter anderem die Zensur. Wenn Dorothea Schlegel etwa an ihren Sohn Johannes über den Kampf gegen Napoleon schreibt, und wenn sie dabei Familienthemen als allegorische Surrogate benutzt, dann will sie damit nicht zuletzt unliebsame Mitleser täuschen - und benötigt dazu Raum. Aber auch die Praxis, nur an einen Adressaten zu schreiben und diesem dann aufzutragen, weitere Freunde und Bekannte in dessen Umgebung, an die kein eigener Brief geschrieben wurde, mit speziellen Informationen zu versorgen, kann den einzelnen Brief anschwellen lassen.

Die hier vorgeschlagene Typologie der Faktoren langer Briefe ist zugleich ein erster Grundriss ihrer Poetik. Sie beansprucht freilich keineswegs, vollständig und erschöpfend zu sein. Vielmehr hat sie ausdrücklich tentativen Charakter. Es ist mehr als wahrscheinlich, dass weitere Faktoren, wie etwa die Frequenz eines jeweiligen Briefwechsels, die Anzahl der an einem Brief beteiligten Schreiber, der räumliche Abstand zwischen Briefverfasserin und -adressat und die soziale Stellung, das Geschlecht sowie das Alter der Korrespondenzpartner die Länge eines Briefes beeinflussen können. Die Einordnung dieser und weiterer Faktoren muss weiteren Forschungen überlassen bleiben.

\section{Lange Briefe in den Briefwechseln Friedrich und Dorothea Schlegels}

Die statistischen Werte, um die es im dritten Teil unseres Beitrags gehen wird, basieren auf einem Gesamtkorpus von knapp 3.000 überlieferten Briefen von und an Friedrich und Dorothea Schlegel, das zwischen 1785 und 1839 entstanden ist. Was die Briefe Dorotheas betrifft, so weisen die insgesamt 309 für diesen Beitrag ausgewerteten Briefe eine Durchschnittslänge von 4.998 Zeichen auf. Das lässt sich der Übersichtstabelle (Tab. 1) entnehmen.

Tab. 1: Brieflänge in Dorothea Schlegels Korrespondenz (1811-1814; 1823-1839; Supplemente).

\begin{tabular}{rrrrrr}
\hline Datenbasis & Kürzester Brief & Kurzer Brief & Durchschnitt & Langer Brief & Längster Brief \\
\hline 309 Briefe von DS & 610 & $<2.500$ & 4.998 & $>10.000$ & 21.268 \\
91 Briefe an DS & 610 & & 4.199 & & 26.351 \\
\hline
\end{tabular}


Entsprechend der Annahme im ersten Teil unseres Beitrags hat also ein langer Brief Dorotheas mehr als 10.000 Zeichen. Etwa ein Fünftel aller Briefe im Korpus hat Dorothea verfasst. Über die Jahre und Schaffensperioden ihres Mannes schwankt der überlieferte Anteil allerdings stark zwischen 10 und 72 Prozent. Ähnliches gilt für die Anzahl langer Briefe.

Berechnet man die ungefähre Durchschnittszeichenzahl jedes einzelnen Bandes der zehnbändigen Ausgabe sämtlicher Briefe von und an Friedrich und Dorothea Schlegel, ${ }^{24}$ so ergibt sich das folgende Diagramm.

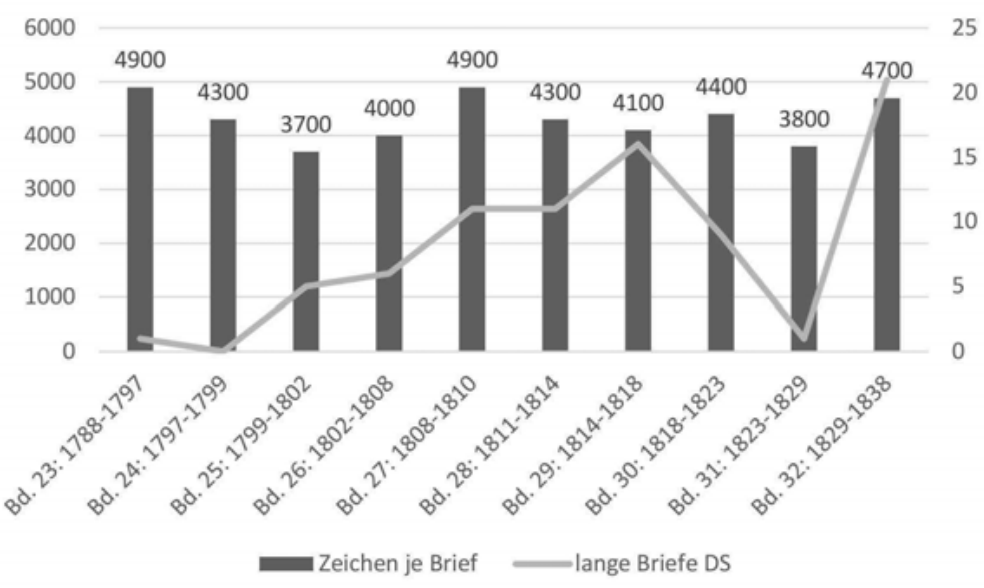

Abb. 1: Durchschnittszeichenzahl Briefbände KFSA.

Es belegt, dass lange Briefe in der Korrespondenz Dorotheas zuerst in der Jenaer Zeit (ab 1799) und dann verstärkt in der ersten Wiener Dekade sowie in den späten Briefen auftreten. Zudem variiert in der Korrespondenz des Ehepaars Schlegel der Brief durchschnittlicher Länge erheblich. Er liegt zwischen 3.700 und 4.900 Zeichen. Die Anzahl langer Briefe (à 10.000 Zeichen) korreliert weder mit der Durchschnittszeichenzahl noch mit den Briefen Dorotheas je Band.

Eine wesentliche schreiberspezifische Ursache für das verstärkte Auftreten langer Briefe dürfte mit Neukonstellationen der Schreibszene Dorothea und Friedrich Schlegels zusammenhängen. Hervorgerufen wurden sie durch politische, soziale oder geographische Veränderungen. Die ersten vier langen Briefe Dorotheas stammen aus dem Jahr 1799 (Band 25) und gehören zur Korrespondenz mit Friedrich

24 Schlegel 1980-. 
Schleiermacher. Das Ausbleiben von Briefen wird von ihr moniert, scheint aber, ebenso wenig wie die gelegentlich von ihr eingestreuten langen Briefe, Störungen im Schreibverhalten veranlasst zu haben. Die hohe Frequenz von 7-14 Tagen je Brief bleibt gleich. Das deutet auf eine von beiden Seiten gezielt herbeigeführte Intensivierung der Beziehung hin. Bestätigen lässt sich das durch den korrespondierenden Umstand, dass umgekehrt kurze Briefe von ihr eigens begründet und entschuldigt werden.

Die Zahl langer Briefe und die Zahl der Adressaten steigt im Fall Dorothea Schlegels mit den Jahren mäßig an, die Frequenz, bezogen auf Briefe durchschnittlicher Länge, sinkt auf 1-2 Briefe pro Monat und weiter auf ein bis zwei Briefe pro Jahr im hohen Alter (nach Schlegels Tod). Zumeist sind die wenigen Briefe einer Korrespondenz nun lange Briefe, die durch familiäre Anlässe wie Geburtsoder Namenstage veranlasst sind. Die Dauer intensiv begonnener Briefwechsel, an deren Anfang oft auch lange Briefe stehen, ebbt nach 1-3 Jahren ab und schläft weitere 1-2 Jahre später oft, aber nicht immer, ein. Ein Beispiel für eine extrem intensive Korrespondenz ist diejenige mit Caroline Pichler. Sie umfasst 23 Briefe, bei denen der Durchschnittswert bei über 10.000 Zeichen liegt. Hier gibt es gewissermaßen nur lange Briefe - was zugleich die Relationalität der Kategorie ,langer Brief‘ verdeutlicht. Ein Beispiel für eine ausgewogene Nähe-Distanz-Beziehung mit einer durchschnittlichen Frequenz von 6-7 Briefen pro Jahr ist die Korrespondenz Dorotheas mit Franz Bernhard von Bucholtz, zu der auch vier lange Briefe gehören. Einer davon wurde für den vierten Teil dieses Beitrags als Analysebeispiel ausgewählt. Mit Bucholtz weiß sich Dorothea Schlegel eins in der Freundschaft zu Friedrich Schlegel sowie in der gemeinsamen Absicht, den Nachlass des Verstorbenen herauszugeben. Zudem gehört Bucholtz zu den wenigen langjährigen Wiener Freunden, die auch Dorothea Schlegels neues Zuhause in Frankfurt und ihre Familie kennen. Der Briefwechsel umfasst eine Zeitspanne von neun Jahren und endet mit Bucholtz' überraschendem Tod.

Zieht man zum Vergleich Dorotheas Ehemann Friedrich Schlegel heran, ${ }^{25}$ so sind seine langen Briefe vor allem an den Bruder gerichtet ( 56 von 85). Eher selten, dann aber ein Beleg für eine Änderung der Beziehungsform, sind seine langen Briefe an Dorothea während ihres Aufenthalts in Rom. ${ }^{26} 14$ weitere lange Briefe gehören zu einem Konvolut von 200 Briefen, die an Christine von Stransky adressiert sind. Die durchschnittliche Frequenz beträgt in diesem Fall 16-17 Tage. Bemerkenswert ist am Schlegel/Stransky-Briefwechsel der Umstand, dass die Frequenz im Laufe der Zeit ansteigt und in den letzten Jahren 1-2 Briefe in der Woche, ja sogar auch

25 Vgl. Patsch 2017.

26 Vgl. Frank 1988, 239. 
einmal 2 Briefe an einem Tag abgesendet werden. Auch hier wird die Störung des Mediums von den Beteiligten nicht als solche erfahren, sondern als Bestätigung der gesuchten Nähe erlebt. Das passt zum Thema dieses Briefwechsels, der sich mit der Seelenverwandtschaft zwischen den Korrespondenzpartnern, verbunden mit Visionen der Freundin und ihrer Ausdeutung durch Schlegel, beschäftigt.

Wie lassen sich die statistischen Befunde nun deuten? Was kann eine philologische Statistik zu einer differenzierten Erkenntnis langer Briefe beitragen? Die folgenden sechs Thesen, die aus der Analyse der Korrespondenz Dorothea Schlegels abgeleitet worden sind, ließen sich vermutlich bei aller gebotenen Vorsicht zumindest für den hier thematischen Untersuchungszeitraum verallgemeinern:

1. Lange Briefe sind seltene Gaben. Das wird unter anderem durch den Umstand bestätigt, dass es sich nur bei 5\% bzw. 9\% der fast 2.400 Briefe des Ehepaars Schlegel um lange Briefe handelt. Einen Liebesbriefwechsel mit Längenexzessen sucht man hier vergebens - wenn auch Dorotheas früher Briefwechsel mit Schleiermacher oder Friedrich Schlegels Korrespondenz mit Christine von Stransky in diese Richtung gehen.

2. Lange Briefe werden nur wenigen auserwählten Freunden oder Familienmitgliedern zuteil. Von den 480 Korrespondenzpartnern des Ehepaars gehören nur 24 Personen zu diesem Kreis.

3. Im Umkehrschluss bedeutet das auch, dass ein neu aufgetauchter langer Privatbrief einer unbekannten Person diese mit hoher Wahrscheinlichkeit als Freund oder Familienmitglied ausweist. Das lässt sich editorisch auch für Zuschreibungsfragen nutzen.

4. Lange Briefe können nicht nur einen Briefverkehr behindern oder zerstören, sondern auch den brieflichen Dialog aufrechterhalten. Das gilt vor allem dann, wenn sie etwa zu wiederkehrenden Festtagen geschrieben und damit ritualisiert werden. Es wäre zu prüfen, ob dieser Typ langer Briefe tatsächlich noch auf eine lange Antwort wartet, oder ob er nicht eher Formmerkmale der Epistel aufnimmt und keine Antwort einfordert.

5. Lange Briefe verlangen weit mehr als Briefe normaler Länge körperliche und geistige Gesundheit, Zeit und Ruhe sowie Anknüpfungspunkte für Erzählbares. Das Fehlen eines oder mehrerer dieser Aspekte wird von Dorothea Schlegel insbesondere in ihren späten Frankfurter Jahren immer wieder zur Sprache gebracht.

6. Nur lange Briefe geben Gelegenheit zu einer bestimmten Form der Konversation, nämlich dem Plaudern oder Schwatzen. Das berührt den zentralen Punkt in der Einschätzung Dorothea Schlegels als Briefschreiberin. Denn hier setzen traditionell die Vorwürfe der Nachwelt an. Für die Zeitgenossen war der Schwatz dagegen durchaus auch positiv konnotiert. In Adelungs Wörterbuch 
heißt es zum Lemma „Schwatzen“ zwar: „einen reichen Fluß der Worte bey unerheblichen Dingen haben“, doch wird dann spezifiziert: „Vertraulich reden, so daß der Nebenbegriff des Unerheblichen verschwindet. [...] Plaudern wird in eben demselben Verstande gebraucht. “27 Man kann das so verstehen, dass die Rede von unerheblichen, alltäglichen Dingen eingesetzt werden kann, um Vertrauen zu signalisieren und zu generieren. Das ist ein spannender Punkt, an dem im Sinne der phatischen Dimension sozialer Austauschprozesse ${ }^{28}$ eine medien- und kulturhistorische Neubewertung der Briefe Dorotheas ansetzen kann. Dorothea, so könnte man sagen, löst das von langen Briefen aufgeworfene medientechnische Problem, indem sie es in einen Vorteil ummünzt: in ein schriftliches Gespräch unter Freunden, das in erster Linie die phatische Seite der Kommunikation bearbeitet und dadurch Vertrauen im Alltag prozessiert. Man muss nur an Dorotheas jüdische Herkunft und an Tiecks Kunstmärchen Der blonde Eckbert denken, um die epochale Brisanz des Verfahrens ermessen zu können. Dorothea Schlegel hat die Kulturpoetik des Schwatzens in einem Brief an Sulpiz Boisserée auch in einem Bild verdichtet und ihren Eigensinn reflektiert - natürlich in einem langen Brief:

Sie sehen ich schwatze noch immer gern allerley durcheinander mit Ihnen, für ein solches freundliches Geschwatz nehmen Sie alles das gesagte, als giengen wir in Ihrem Blumen Garten herum, und raisonnirten über unsre Liebhaberei für diese und jene Blume, weiter bedeutet alles das Nichts [...].29

\section{Beispielanalyse}

Im letzten Teil geht es um die exemplarische Analyse eines langen Briefes. Dafür wurde der Brief ausgewählt, den Dorothea Schlegel am 16. und am 27. November 1830 an Franz Bernhard von Bucholtz geschrieben hat. Bucholtz, österreichischer Diplomat und katholischer Publizist aus Münster, war seit 1813 mit Friedrich Schlegel und in der Folge auch mit seiner Frau Dorothea bekannt und befreundet. Bucholtz und Schlegel arbeiteten 1816 bis 1818 gemeinsam in Frankfurt für die österreichische Gesandtschaft. Nach Friedrich Schlegels Tod blieb zwischen ihm und der verwitweten Dorothea bis zu Bucholtz' Tod im Jahr 1838 eine enge Freundschaft bestehen, die sich in Folge von Dorotheas Umzug nach Frankfurt am

27 Adelung 1798, 1725.

28 Vgl. Stanitzek 2001.

29 Dorothea Schlegel an Sulpiz Boisserée, Wien, 16. Febr. 1811, Köln, HAK, Best. 1018 (Boisserée, Sulpiz), A 297, Nr. 28a. 
Main im Jahr 1830 vor allem in wechselseitigen Briefen niedergeschlagen hat. Auf diese Korrespondenz wurde im dritten Teil des Beitrags bereits eingegangen.

Die folgende Analyse des ausgewählten Briefs wird von einer doppelten Fragestellung geleitet: Inwiefern zeigen sich in ihm typische Merkmale langer Briefe und in welcher Weise entschärft er das Störpotential langer Briefe? Was die erste Frage betrifft, so finden sich gleich mehrere typische Ursachen des langen Briefs. Bezogen auf die Schreibszene wird deutlich, dass die Schreiberin durch „Unpäßlichkeiten“ (Z. 13) zunächst am Schreiben gehindert war und dass sie erst wieder gesunden musste, um die „Anstrengung“ (Z. 16) des Schreibens auf sich nehmen zu können. Als sie dann einmal in Fahrt war, hat „eine reiche Sendung aus Wien“ ihr „Briefe von allen lieben Freunden“ gebracht (Z. 96-97), die sie sofort verschlungen hat. Das hat die Fortsetzung des Briefes elf Tage lang herausgezögert (Z. 98-99). Bezogen auf das Dokument selbst gibt die Schreiberin an, dass sie den Brief an Bucholtz über die österreichische Gesandtschaft und nicht über die Post expedieren will. Aus beförderungstechnischen Gründen muss sie sich deshalb „nicht mit dem Raum geniren“ (Z. 100-101), kann also länger und ausführlicher schreiben. Zu den adressatenbezogenen Aspekten gehört schließlich der Umstand, dass Bucholtz von Dorothea als eine Art Relaisstation innerhalb von Wien adressiert wird, die Grüße und Nachrichten an eine Reihe von namentlich benannten Personen weiterleiten soll. Auch der paradoxe Umstand, dass Dorothea zum Schreiben mehrerer Briefe an viele Adressaten offenbar die Kraft fehlt, steigert - gewissermaßen auf Kosten nichtgeschriebener anderer Briefe - die Länge dieses einen und belegt überdies die zentrale Stellung, die der Adressat in ihrem Wiener Bekanntenkreis einnimmt, sowie den hohen Grad an Vertrauen zu ihm.

Das leitet über zur zweiten Frage, die den Umgang der Schreiberin mit dem Störungspotential langer Briefe betrifft. Grundsätzlich lässt sie keinen Zweifel daran, dass sie an den Wiener Verhältnissen brennend interessiert ist und daher überaus gerne „einen langen langen, ausführlichen Brief“ (Z. 54) erhalten würde - und sei es von entfernten Bekannten. Auch wird deutlich, dass Dorotheas zunehmende Mühe mit dem Briefeschreiben ihr den Vorwurf einhandeln kann, die Wiener Freundinnen und Freunde mit allzu „sparsamen Worten“ (Z. 22) abzuspeisen. Das will sie um jeden Preis vermeiden. Sie versucht also, mit dem einen Brief an Bucholtz möglichst viele weitere Adressaten mitzubedienen. Das macht den Brief wie gesagt lang, schlägt sich aber auch in seiner Struktur nieder. Nachdem sie dem Adressaten ein wenig gerührt den Anfang von Friedrich Schlegels Lied Huldigung zitiert hat, unterbricht sie sich, um sich zur Ordnung zu rufen: „Ich muß endlich zu schwatzen aufhören, denn ich habe noch gar viele Briefe zu beantworten vor mir.“ (Z. 151-152)

Schaut man sich den Brief an Bucholtz unter dem Aspekt des Schwatzens oder Plauderns an, dann fällt auf, dass sie die Tendenz zum Monologischen, die dem langen Brief innewohnt, ebenso geschickt wie gezielt vermeidet. Zum einen bietet 
sie ihre Nachrichten in Form von kleinen anekdotischen Erzähleinheiten dar, die sie häufig durch Gedankenstriche voneinander absetzt. Das folgt der „Poetik der Digression“, die durch Gellert in die Briefpraxis eingeführt worden ist, ${ }^{30}$ und erinnert an die Praxis des Fragmentierens, die Friedrich Schlegel in der Anfangsphase seiner Beziehung zu Dorothea zur Kunst erhoben und Dorothea Schlegel vor allem in ihrem Tagebuch auch selbst praktiziert hat. ${ }^{31}$ Der Brief nähert sich insofern thematisch dem Klatsch und steht formal den kleinen und vermischten Nachrichten nahe, wie sie dann für die Zeitung typisch werden. Zum anderen hält die Schreiberin den Adressaten durch Indexikalisierung ständig präsent. Zu diesem Zweck verwebt sie die Personalpronomen der 1. und 2. Person Singular auf gekonnte Weise und erzeugt insofern mit genuin literarischen Mitteln ein Analogon der sozialen Praxis des Plauderns. Folgt man der These Vellusigs, ${ }^{32}$ nach der sich im Brief des 18. Jahrhunderts ein „zuerst im Frankreich des 17. Jahrhunderts kultivierte[s] Stilideal nonchalanter Leichtigkeit“ etabliert, das aus „der Konversationskultur der Salons, die von Frauen dominiert wurden“, stammt, dann fällt es nicht schwer, in der angeblichen Geschwätzigkeit der langen Briefe Dorothea Schlegels die urbanen Strukturen der frühromantischen Berliner Salonkultur wiederzufinden, ${ }^{33} \mathrm{zu}$ der sie einen eigenständigen Beitrag geleistet hat. ${ }^{34}$ In Schleiermachers Theorie des geselligen Betragens ${ }^{35}$ hat sie ihr intellektuelles Monument gefunden und im gemeinsamen Einsatz Dorotheas, Rahel Varnhagens und Sophie Schlossers für die Ausbildung katholischer Mädchen während Dorotheas Aufenthalts in Frankfurt $^{36}$ sowie in der ,Frauenkommune‘, deren Zentrum Dorothea während ihres Aufenthalts in Rom bildete, ist sie unter dem Aspekt eines im weitesten Sinne karitativen Engagements wiederaufgenommen worden. ${ }^{37}$ Das wäre künftig genauer zu untersuchen. Lange Briefe könnten dabei behilflich sein.

30 Vellusig 2000, 104-105.

31 Stern 2006, 217.

32 Vellusig 2000, 100.

33 Nach Stern $(2006,71)$ gilt mit Blick auf den emanzipatorischen Charakter dieser von jüdischen Frauen initiierten Gesellschaftsform: „In ihren Salons erstrebten sie Gegenseitigkeit in den Beziehungen zwischen Frauen und Männern, einen wechselseitigen Austausch von Fähigkeiten und Erfahrungen, Gefühlen und Energien.“ Das sind zugleich wesentliche Bestimmungsmomente der romantischen Briefkultur.

34 „Durch diese Salonkultur ist Brendel Veit nachhaltig beeinflußt worden; sie hat die entscheidende Wende ihres Lebens mit herbeigeführt.“(Stern 2006, 69) Zu Dorotheas eigenem Salon vgl. Schoeps (2020, 10-11).

35 Schleiermacher 1984.

36 Vgl. Frank 1988, 243.

37 Vgl. Ujma 2001, 53. 


\section{Fazit}

Der Beitrag stellt das Medienformat des langen Briefs als ernst zu nehmenden Gegenstand der Literaturwissenschaft und insbesondere der Briefforschung vor. Er bietet dazu eine erste Typologie an und formuliert am Beispiel eines größeren Briefkorpus sechs statistisch belegte Thesen zum langen Brief. Schließlich wurde die Leistungsfähigkeit des hier gewählten medienwissenschaftlichen Ansatzes an einem Beispielbrief überprüft. Die zentrale These des Beitrags lautet, dass das mathematisch gut erfassbare Merkmal der Brieflänge etwas über Nähe und Distanz in Briefwechseln verrät und dass zu dessen Erklärung eine philologische Statistik des Briefes geeignet erscheint. Sie ließe sich unschwer mit rezenten Interessen der Digital Humanities verbinden, bietet aber über das Konzept der ,störenden Gabe“ auch Anschlussmöglichkeiten an eine Kulturanthropologie des Briefes.

\section{Anhang}

Dorothea Schlegel an Franz Bernhard von Bucholtz ${ }^{38}$

Frankfurt am Main, Dienstag, 16. bis Samstag, 27. November 1830

Frankf: $16^{\text {ten }}$ Nov: 30 .

Sie haben mir rechte Freude gemacht lieber Freund Bucholtz durch Ihren schönen Brief, in der Begleitung eines Schreibens Ihrer lieben Frau Mutter, welches mich um so mehr freute, und überraschte, da ich durchaus ohne Nachricht von dersel-

5 ben war, und in manchen hypochondrischen Momenten mir wohl einbildete, sie sey längst durch Frankfurth durchgereißt, ohne mich aufgesucht, oder aufgefunden zu haben! Einige Tage vor Ihrer Sendung kam ein Brief von Klinkowström, der nach der langen Zeit, in welcher ich kein Wort von Wien vernommen hatte, mir ungemein tröstlich gewesen war, jedoch enthielt er, obgleich mit lebhaften Freundschaftsbezeugungen, nur spärliche Nachrichten, von Ihnen und Ihrer Frau Mutter z. B: war nichts darin enthalten, und so wissen Sie jetzt genau, wie so Ihr Brief mir doppelt und dreyfach angenehm war. Ich würde ihn auf der Stelle mit einem Andern erwiedert haben, allein eine meiner gewöhnlichen Unpäßlichkeiten hielt mich einige Tage von jeder Beschäftigung ab; auch noch bin ich nicht ganz 15 wieder im Geschick, und noch den Verordnungen des Arztes anheim gefallen, der

38 Wir danken Cosima Jungk für die Zweitkorrektur des Briefes. 
jede Anstrengung untersagt. Sollte ich also dieses mal nicht im Stande seyn, Ihrer Frau Mutter, meiner lieben Franzisca, der Hofräthin Müller, und an Pilat, jeden besonders zu schreiben, (wie es allerdings in meiner Intention ist) so bitte ich Sie, um Ihrer gewohnten Freundschaft willen, allen Allen lieben Freunden, meine innigsten besten Grüße melden zu wollen. Möchten sie doch ja nicht, mein immer währendes Andenken, mein Dankgefühl in Erinnerung ihrer Liebe, nicht nach meinen sparsamen Worten abmessen! Gewiß, im Gegentheil, die Entferntesten, sind mir gewöhnlich die Nächsten! - Besonders bitte ich Sie dann mich bey Frau v. Pichler zu entschuldigen, daß ich ihr noch nie geschrieben, ich komme nur langsam herum, um jedem zu schreiben, während ich von Allen recht leicht öfters etwas höhren könnte. Meine Familie ist seit einem vollen Monathe schon hier, durch Gottes Hülfe recht fröhlich und gesund angelangt; es giebt viel zu thun, so viel, daß ich vielleicht gezögert haben würde die Sorge zu übernehmen, wenn ich nach meiner gewöhnlichen Unvorsichtigkeit, nicht alle Schwierigkeiten unbeachtet, und unerkannt gelassen hätte. Es ist aber besser so; ich bin | recht froh, mir sagen zu dürfen, daß ich nicht vergeblich, nicht um meines Vergnügens halber hier bin, sondern daß ich im Gegentheil recht nothwendig u nützlich zu seyn die Ueberzeugung habe. Ich bitte alle meine lieben Geistes und Gebeths verwandten mich mit ihrem Gebethe zu unterstützen, daß mir Gott Kraft und Ausdauer verleihe, so lange es noth thut. Es sind fünf allerliebste Kinder, gut geartet, einfach u natürlich, von dieser Lebhaftigkeit aber kann man sich nicht leicht eine Vorstellung machen, was bey der gänzlichen Unbekanntschaft mit unsrer Sprache, den Umgang mit ihnen erschwert, und eine gewisse Methode der Erziehung unpraktikabel macht. Der Tod der Julie Bruchmann hat mich tief erschüttert; das ist eine sehr wunder-

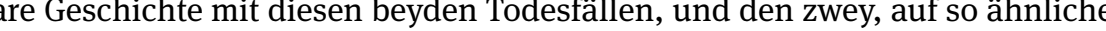
Art als Waisen zurückgelassenen Kindern der beiden jungen Mütter. Ein gewöhnliches Bedauern und Trauern, dünkt mich, ist hier nicht hinreichend, es ist etwas mehr als in dem gewöhnlichen Gang der Begebenheiten vorkommendes darin! ich möchte etwas darum geben den Wittwer zu sprechen. Schreiben kann ich ihm ihn - Der Hofräthin Müller würde ich gern schreiben, wenn ich dazu käme; ich denke recht viel an sie, und wie unser Schicksal sich immer wieder verschwistert zeigt. Hatte sie nicht, wie auch ich, nichts so sehr im Sinn, als immer mehr sich zurück zu ziehen, immer stiller und einsamer zu werden? und ist sie nicht, eben wie ich, dem Herzens Wunsche entgegen, genöthigt eine große Haushaltung zu führen, und sich mit vielen Menschen umgeben zu sehen, und wieder aufs Neue Alle Art von Sorgen, liebend zu über nehmen? Tausend Grüße, der Mutter und den Töchtern, eine von ihnen könnte wohl so liebenswürdig seyn mir wieder einmal einen langen langen, ausführlichen Brief zu schreiben, wo recht viele Nahmen darin vorkommen, ich möchte so gern recht viel lesen u erfahren, was mir wichtig 
$\mathrm{u}$ werth ist; daß ich den lieben Mädchen eine Antwort schuldig geblieben bin sollten sie doch aus christlicher Liebe nicht so genau nehmen, auch wird ihnen das mittheilen leichter als mir; mir sind Personen, und Umstände bekannt, aber wo sollte ich anfangen sie mit meinen für sie neuen Verhältnissen, $u$ Umgebungen intek interessiren? Dasselbe gilt auch für Franzisca, doch hoffe ich ihr bald zu schreiben, die Römerin ist ihr nicht so unbekannt. - Meiner theuern Schwester geht es Gott sey Dank wieder gut; nach den neuesten Berichten, geht sie wieder, wenn auch unterstützt | im Zimmer umher, und sie hat mir eigenhändig einen recht langen

65 muntern Brief geschrieben, mit ihrer gewöhnlichen festen Handschrift. Ach Gott wolle doch ferner diesen schrecklichen Unfall von ihr abwenden; zu traurig ist es, sich immer vor der Rückkehr desselben fürchten zu müßen; welche schöne liebe Tage habe ich zu Dresden in Gesellschaft der theuern Seele verlebt; sie werden mir unvergesslich bleiben, sie gehörten ohne Zweifel zu den Schönsten meines Lebens. 70 - Vom Abbé K. habe ich, so wie Sie, einen Brief aus Paris erhalten, mit der hiebey folgenden Einlage die Sie wohl so gütig sind, bey Gelegenheit zu übergeben. Ich beantwortete seinen Brief bald nach dessen Empfang, und kann Ihnen nun nicht versprechen, ihm so bald wieder zu schreiben. Sollte ich aber durch irgend etwas dazu aufgefordert werden, soll Ihr Auftrag auch gleich an ihn befördert werden.

75 Natürlicher hätte mir geschienen, Sie hätten einige Zeilen direct an ihn gerichtet, und diese mir zur weitern Besorgung überschickt. - Nach den Aeußerungen seines Briefes, der ziemlich verworren, und sogenannt mystisch abgefasst ist, so hat ihn sein Herr und Meister auf's Land, in der Bretagne in die ordentliche Seelsorge geschickt, welches er als ein summum sacrificium anzusehen scheint, mir aber als das ganz, und einzig rechte für den armen Abbé dünkt. Vielleicht daß er in dieser Thätigkeit wieder festen Boden unter seinen Füßen gewinnt. Sie haben doch wohl in W. l'Avenir? Dies Journal nämlich welches Abbé Lamenais jetzt redigirt, und worin sehr geistreiche, merkwürdige Aufsätze, von ihm selbst, und von mehreren Andern z. B. Robjano Boorsbek etc, enthalten sind? Haben Sie es noch nicht, so 85 schaffen Sie es nur gleich an: Es ist die Wendung, an welcher Sie wohl bis jetzt noch nicht gedacht, nämlich das Anschließen, um nicht Vereinigung zu sagen, des ächten, ungebognen Katholizismus, an den unbegränzten Freyheits Liberalismus, mit donnernder und blitzender Beredsamkeit, und einen alles fortziehenden Witz. Meinem eignen Gefühl nach, ist es der Ton der auch darin niederschlägt, und mir

90 für einen geistlichen Wortführer ungeziemend scheint; in den Meinungen selbst, so wie an seine Forderung derselben unbegränzten Freyheit für die Kirche, wie für jedes Andre im Staat bestehende, muß man aber trotz des etwas rauh heraus wehenden Windes, sich wohl anschließen, u Respekt haben. Lesen Sie es nur, und schreiben Sie mir zu meiner Belehrung, und Aufklärung, Ihre Meinung, und das Urtheil der Geistlichen in W. | 
27ten Nov: So weit war ich gekommen, als eine reiche Sendung aus Wien, Briefe von allen lieben Freunden, mir zugestellt wurde, und ich vor lauter lesen nicht gleich wieder zum weiter schreiben kam - und da ich nun überzeugt wurde, daß meine erste Sendung durch die Gesandschaft nicht verlohren ist, wie ich befürchtete, so will ich nur gleich mich nicht mit dem Raum geniren, was ich mit Post schreibend, wohl gethan haben würde, und will Ihnen noch allerley sagen und fragen; einmal aber im schreiben unterbrochen kam ich bis heute nicht dazu. Wie es scheint kann Ihre Frau Mutter nicht recht zum Entschluß kommen, ob sie in Wien bleiben oder zu ihrer Tochter zurück gehen soll? ich kann mir das lebhaft 105 vorstellen; es wird mir nicht besser gehen, wenn ich mich werde entscheiden sollen, wieder nach Wien zurück, wohin so manches mich zieht, oder hier bey den Meinigen zu bleiben, wo mich nicht weniger hält, oder einen Besuch in Berlin zu machen, wohin meine Schwester mich ruft?! wenn nicht wieder eine Entscheidung aus des Himmels Höhen kommt, so wie im vorigen Sommer Sie ganz unvermuthet hinzutraten mich zu entscheiden, so wird es auch mit mir nicht leicht zu einem resultat kommen. - Lassen Sie mich doch nicht die Letzte seyn, die erfährt was Ihre Frau Mutter beschließt? Es scheint wohl als gefiele es ihr nicht übel in Wien, aber wundern würde es mich nicht, wenn sie auch wohl manchmal sich nach ihrem gewohnten Hausbestand zurück wünscht; der Mensch ist ja einmal so daß er sich der Beschränkung des irdischen Lebens nicht gern unterwerfen mag; und das wird immer ärger, je mehr man sieht und kennen lernt. Von Dorothea Tieck habe ich einen sehr lieben schönen Brief, worin sich ihre heitre ruhige Fassung, und ihr klarer Geist, so wie ihre liebefähige Seele sich in sehr einfachen Worten, ohne alles Gesuchte, oder Prunkhafte ausspricht. Es ist mir nicht leicht eine Person vorgekommen die so viel Bildung, mit solcher Anspruchlosigkeit, so viel Frömmigkeit mit so vielen Verstand verbindet.

Ich wünsche oft Sie hier zu haben lieber Freund, Ihr Gespräch, Ihre Ansichten, und überhaupt Ihr freundschaftlicher Umgang, das ist es was uns hier, bey manchem Guten recht empfindlich fehlt. Mit Philipp würden Sie zufrieden seyn, er hat bedeutend | seit Sie ihn in Rom gesehen haben, an ruhige besonnene Kraft, und an Bestimmtheit der Denkungsart; seine Arbeiten sind recht schön, und sprechen alle mehr, oder weniger jenen Bedeutsamkeit der klaren religieusen Gesinnung aus, von welcher er ganz durchdrungen ist; sein Umgang ist leicht, gefällig, nachgebend, und er weiß sich unter den Hiesigen sehr verschieden Denkenden, allgemeine Liebe und Achtung zu erwerben, so daß sein Standpunkt hier beym Institut, der für viele Andre sehr dornig seyn könnte, ihn recht zufrieden seyn läßt, nur daß ihm mehr Zeit geraubt wird, als er gern hergiebt. Ein gewaltiger Jäger vor dem Herrn, ist er aber doch noch immer, und dazu wird er hier immer mehr verleitet, was mir eigentlich nicht lieb ist. - Schlossers sind immer noch nicht wieder hier, und 
135 werden wohl nicht leicht vor Weynachten kommen. Für mich recht unangenehm, ich vermisse sie allenthalben. Senator Vogt habe ich schon einige mal gesehen; der hat sich doch gar nicht verändert. Im Avenir hat vor einigen Tagen ein Artikel über den Zustand in Deutschland gestanden, haben Sie ihn gelesen? und was meynen Sie dazu? scheint es Ihnen nicht kränkend sich so etwas sagen lassen zu 140 müßen, ohne dreist erwiedern zu dürfen tu en a menti! - Es ist eine sonderbare Erscheinung dieses Avenir. -

Denken Sie jetzt nicht oft an den sellgen Friedrich, bey den Begebenheiten unsrer Zeit? hat er wohl zuviel gefürchtet, wenn er den Umsturz Alles Bestehenden voraussah?

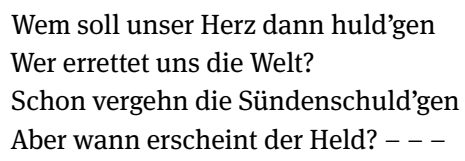

Ich erinnerte neulich meine Schwester an diesem schönen Liede, und seitdem kömmt es mir nicht mehr aus dem Sinne.

Ich muß endlich zu schwatzen aufhören, denn ich habe noch gar viele Briefe zu beantworten vor mir. Sind Sie so gütig gewesen, bey Schaumburg ein Exemplar der letzten Vorlesungen für den Prof: Clar nach Prag senden zu lassen? und wie sind Ihre Hoffnungen für die Fortsetzung der sämmtlichen Werke? Tieck machte mir damals recht gute Vorschläge | wollen Sie nicht sich mit ihm darüber in Correspondenz setzen? Der Buchhändler Winter hat mir wieder einen unangenehmen Mahn Brief geschrieben, und mir gedroht mich zu verklagen, aber ich weiß nicht ob er das kann, doch drückt mich seine Forderung. Werden Sie mir nicht ein Exemplar Ihres Ferdinands zuschicken? Darauf rechne ich mit Bestimmtheit.

160 Gott sey mit Ihnen, erfreuen Sie bald wieder durch einen Brief Ihre Freundin

\section{Dorothea Schlegel}

Meine herzlichsten Grüße Ihrer theuern Frau Mutter ich hoffe sie verzeyht mir, daß ich nicht besonders an sie schreibe. Meine Schwester hat mir dankbare Empfehlungen für sie, wie für Ihnen aufgetragen; ihre gütige Theilnahme hat sie sehr gerührt.

Noch ein Wort. Das Güntherische Werk ist hier von den Sachverständigen mit Beyfall und Interesse aufgenommen z. B. von Görres, Brentano., Steingaß, Schwiegersohn des Görres der hier die Vaccanzen zugebracht, und den ich oft gesehen habe. Den humoristischen Theil dieses Werks, welchen man einstimmig dem P. 
170 Veith zuschreibt wollte man nicht goutiren, man findet die Ideen darin aus der Mode, und veraltet, und den Styl manierirte Nachahmung Jean Pauls, Tiecks u sw. was ist denn das für ein Missverständniß mit Damison, und wer ist dieser Damison? Ich gratulire zur Fürstin Metternich.

Philipp sendet tausend Grüße

175 wollen Sie die Güte haben die Einlage an Frau Clar zur Post zu geben?

H: Münster, LAV NRW, V 503 Nachl. F.B. Bucholtz, 305 (18) - 1 DBl. 1 Bl. 6 S., Tinte, Seitenmaß: $206 \times 238 ; 212 \times 261$, D: ungedruckt.

\section{Literatur}

Adelung, Johann Christoph (1811). „Schwatzen“, in: Grammatisch-kritisches Wörterbuch der hochdeutschen Mundart. Bd. 3. Leipzig: 1723-1724.

Brinkmann, Rolf Dieter (1979). Rom, Blicke. Reinbek b. Hamburg.

Bürgel, Peter (1979). „Brief“, in: Kritische Stichwörter zur Medienwissenschaft. Hg. v. Werner Faulstich. München: 26-47.

Deibel, Franz (1905). Dorothea Schlegel als Schriftstellerin im Zusammenhang mit der romantischen Schule. Berlin.

Finke, Heinrich (1918). Ueber Friedrich und Dorothea Schlegel. Köln.

Frank, Heike (1988). „... die Disharmonie, die mit mir geboren ward, und mich nie verlassen wird ... “. Das Leben der Brendel/Dorothea Mendelssohn-Veit-Schlegel (1764-1839). Frankfurt a. M., Bern, Paris u. a.

Fricke, Harald (2007). „Erkenntnis- und wissenschaftstheoretische Grundlagen“, in: Handbuch Literaturwissenschaft. Hg. v. Thomas Anz. Bd. 2. Stuttgart u. Weimar: 41-54.

Kemper, Dirk (1997). „Epistel“, in: Reallexikon der deutschen Literaturwissenschaft. Bd. 1. Hg. v. Klaus Weimar, Harald Fricke u. Jan-Dirk Müller. Berlin u. New York: 473-475.

Körner, Josef (21969). Krisenjahre der Frühromantik. Briefe aus dem Schlegelkreis. Hg. v. Josef Körner. Bd. 2. Bern u. München.

Meyer, Richard M. (1907). „,Vollständigkeit‘. Eine methodologische Skizze“, in: Euphorion 14: $1-17$.

Nickisch, Reinhard M. G. (1991). Brief. Stuttgart.

Patsch, Hermann (2017). „Briefe“, in: Friedrich Schlegel-Handbuch. Leben - Werk - Wirkung. Hg. v. Johannes Endres. Stuttgart: 280-290.

Rilke, Rainer Maria (1996). „Herbsttag“, in: Werke. Kommentierte Ausgabe in vier Bänden. Hg. v. Manfred Engel, Ulrich Fülleborn, Horst Nalewski und August Stahl. Bd. 1. Frankfurt a. M. u. Leipzig: 281.

Schlegel, Dorothea (1881). „Aus Dorotheas Tagebuch“. Dorothea v. Schlegel, geb. Mendelssohn und deren Söhne Johannes und Philipp Veit. Briefwechsel. Hg. v. Johann Michael Raich. Bd. 1. Mainz. 
Schlegel, Friedrich (1980-). Kritische Friedrich Schlegel Ausgabe. Hg. v. Ernst Behler, fortgef. von Andreas Arndt, ab 2007 hg. v. Ulrich Breuer. Abt. III: Briefe von und an Friedrich und Dorothea Schlegel. Bde. 23 bis 32. Paderborn.

Schleiermacher, Friedrich Daniel (1984). „Versuch einer Theorie des geselligen Betragens“, in: Kritische Gesamtausgabe. Bd. 2. Hg. v. Günter Meckenstock. Berlin u. New York: 165-184.

Schoeps, Julius H. (2020). Dorothea Veit/Schlegel. Ein Leben zwischen Judentum und Christentum. Berlin u. Leipzig.

Schupp, Johann Balthasar (1665). Der ungeschickte Redner. Übers. v. Balthasar Kindermann. Wittenberg.

Spoerhase, Carlos u. Nikolaus Wegmann (2018). „Skalieren“, in: Historisches Wörterbuch des Mediengebrauchs. Hg. v. Heiko Christians, Matthias Bickenbach u. Nikolaus Wegmann. Bd. 2. Köln, Weimar u. Wien: 412-424.

Spoerhase, Carlos (2018). Das Format der Literatur. Praktiken materieller Textualität zwischen 1740 und 1830. Göttingen.

Stach, Rainer ( $\left.{ }^{6} 2015\right)$. Kafka. Die Jahre der Entscheidungen. Frankfurt a. M.

Stanitzek, Georg (2001). „Kriterien des literaturwissenschaftlichen Diskurses über Medien“, in: Mediensprache. Medienkritik. Hg. v. Ulrich Breuer u. Jarmo Korhonen. Frankfurt a. M.: 357-384.

Stern, Carola (132006). „Ich möchte mir Flügel wünschen“. Das Leben der Dorothea Schlegel. Reinbek b. Hamburg.

Vellusig, Robert (2000). Schriftliche Gespräche. Briefkultur im 18. Jahrhundert. Köln, Weimar u. Wien.

Ujma, Christina (2001). „Briefe aus der römischen Freiheit - Dorothea Schlegel in Italien“, in: Harmony in Discord. German Writers in the Eighteenth and Nineteenth Centuries. Hg. v. Laura Martin. Oxford: 45-66. 
